\title{
Gas Sensors Based on Tin Oxide Nanoparticles Synthesized from a Mini-Arc Plasma Source
}

\author{
Ganhua Lu, ${ }^{1}$ Kyle L. Huebner, ${ }^{2}$ Leonidas E. Ocola, ${ }^{3}$ Marija Gajdardziska-Josifovska, ${ }^{4}$ and Junhong Chen ${ }^{1}$ \\ ${ }^{1}$ Department of Mechanical Engineering and Laboratory for Surface Studies, University of Wisconsin-Milwaukee, Milwaukee, \\ WI 53211, USA \\ ${ }^{2}$ Department of Mechanical Engineering, University of Wisconsin-Milwaukee, Milwaukee, WI 53211, USA \\ ${ }^{3}$ Center for Nanoscale Materials, Argonne National Laboratory, Argonne, IL 60439, USA \\ ${ }^{4}$ Department of Physics and Laboratory for Surface Studies, University of Wisconsin-Milwaukee, Milwaukee, WI 53211, USA
}

Received 15 March 2006; Revised 20 July 2006; Accepted 1 August 2006

Miniaturized gas sensors or electronic noses to rapidly detect and differentiate trace amount of chemical agents are extremely attractive. In this paper, we report on the fabrication and characterization of a functional tin oxide nanoparticle gas sensor. Tin oxide nanoparticles are first synthesized using a convenient and low-cost mini-arc plasma source. The nanoparticle size distribution is measured online using a scanning electrical mobility spectrometer (SEMS). The product nanoparticles are analyzed ex-situ by high resolution transmission electron microscopy (HRTEM) for morphology and defects, energy dispersive X-ray (EDX) spectroscopy for elemental composition, electron diffraction for crystal structure, and X-ray photoelectron spectroscopy (XPS) for surface composition. Nonagglomerated rutile tin oxide $\left(\mathrm{SnO}_{2}\right)$ nanoparticles as small as a few nm have been produced. Larger particles bear a core-shell structure with a metallic core and an oxide shell. The nanoparticles are then assembled onto an e-beam lithographically patterned interdigitated electrode using electrostatic force to fabricate the gas sensor. The nanoparticle sensor exhibits a fast response and a good sensitivity when exposed to $100 \mathrm{ppm}$ ethanol vapor in air.

Copyright (c) 2006 Ganhua Lu et al. This is an open access article distributed under the Creative Commons Attribution License, which permits unrestricted use, distribution, and reproduction in any medium, provided the original work is properly cited.

\section{INTRODUCTION}

Rutile tin oxide $\left(\mathrm{SnO}_{2}\right)$, a wide band gap (3.6 eV at $\left.300 \mathrm{~K}[1]\right)$ $n$-type semiconductor material, is widely used as sensing elements in gas sensors [2]. The sensing mechanism is based on the fact that the adsorption of oxygen on the semiconductor surface can cause a significant change in the electrical resistance of the material [3]. The formation of oxygen adsorbates $\left(\mathrm{O}_{2}^{-}\right.$or $\left.\mathrm{O}^{-}\right)$results in an electron-depletion surface layer due to electron transfer from the oxide surface to oxygen [4]. Recent studies [5, 6] have shown that the use of tin oxide nanocrystals as sensing elements significantly improves the response and the sensitivity of sensors since the space charge region may develop in the whole crystallite.

Tin oxide nanoparticles have been produced by both colloidal and aerosol routes. The colloidal synthesis route affords considerable control over particle size and structure since the surface chemistry can be manipulated through adjustment of the solution properties [7-9]. Nanoparticles produced in the gas phase can be subsequently deposited onto solid substrates for immediate device applications. Aerosol routes hence provide more flexibility in process control
$[10,11]$ and improve the compatibility of the nanoparticle sensor fabrication process with existing microelectronics fabrication facilities. In addition, the higher processing temperature employed in aerosol synthesis facilitates production of stable phases that are difficult to achieve in colloidal synthesis [12].

This paper introduces a simple, convenient, and low-cost mini-arc plasma source to synthesize tin oxide nanoparticles at atmospheric pressure. Because of the small crystallite size, high resolution transmission electron microscopy (HRTEM) becomes a powerful technique to analyze the product nanoparticles. The new source shows great potential in producing high-quality tin oxide nanoparticles for gas sensing applications. A miniaturized gas sensor has been fabricated using the as-produced tin oxide nanoparticles. The microfabricated nanoparticle sensor exhibits good sensitivity and dynamic response to low concentration ethanol gas.

\section{EXPERIMENTAL DETAILS}

A schematic diagram of the nanoparticle synthesis system, including the mini-arc plasma reactor and devices for 


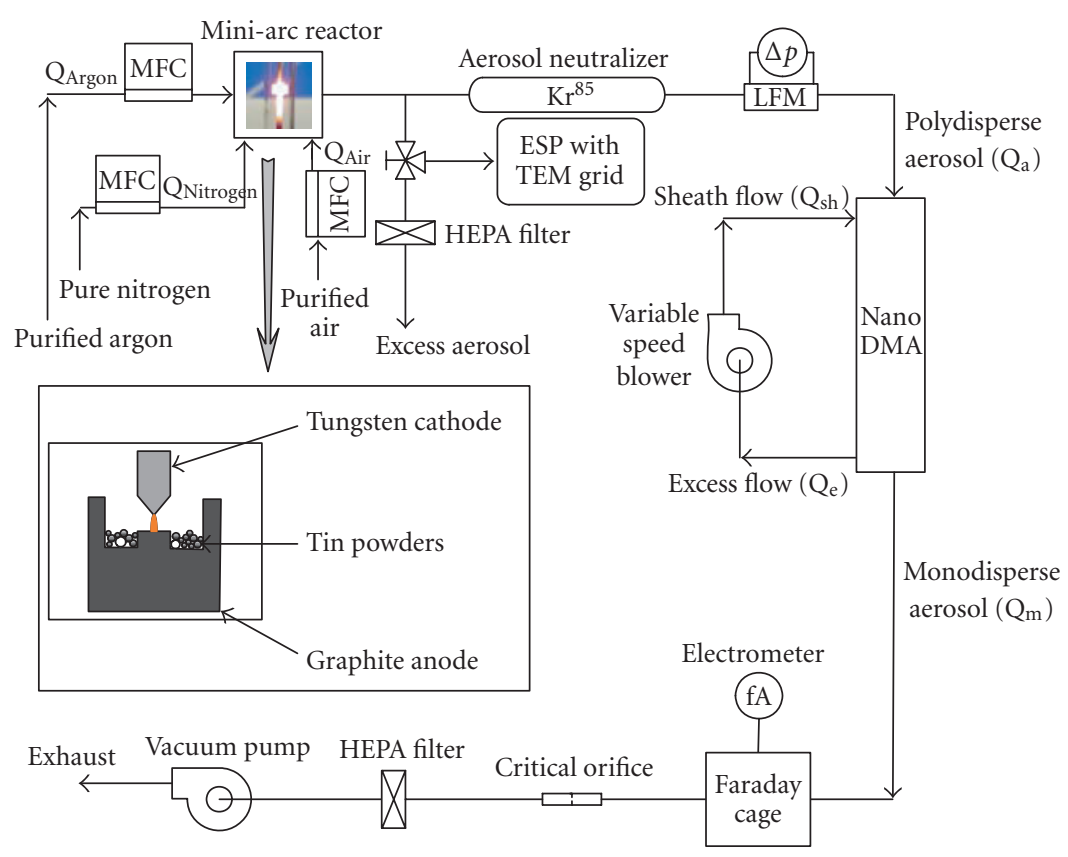

Figure 1: Schematic diagram of the experimental setup for nanoparticle synthesis using a mini-arc plasma source. The inset shows the magnified cathode and anode loaded with tin powders.

collection and characterization of nanoparticles, is shown in Figure 1. The atmospheric mini-arc reactor consists of a $1 / 16^{\prime \prime}$ tungsten rod cathode and a $1 / 4^{\prime \prime}$ graphite rod anode housed in a chamber constructed with Swagelok fittings and quartz tube. The cathode tip was sharpened to facilitate the arc initiation. The anode was designed to maximize the utilization of thermal energy from the arc discharge. To facilitate the arc stability, the anode surface was machined to form an annulus groove so that the $\mathrm{dc}$ arc only burns between the cathode tip and the central island on the anode surface. The typical discharge gap was on the order of $0.1 \mathrm{~mm}$, and it may be adjusted using a translation stage on which the anode was mounted. Coarse tin powders or small pieces $(\sim 1 \mathrm{~mm})$ of solid tin cut from commercially available pure tin wires were placed in the groove between the central island and the outside wall of the graphite anode. A commercial tungsten inert gas (TIG) arc welder (Miller Maxstar 150 STL) was used to drive the $\mathrm{dc}$ arc. Purified argon was used as the plasma and carrier gas. The high temperature in the arc discharge melts and vaporizes the solid tin from the graphite crucible. A pure and cold nitrogen flow was injected to quench the tin vapor and nucleate tin nanoparticles, which were then oxidized to form tin oxide nanoparticles by introducing purified air immediately at the exit of the mini-arc reactor.

The product tin oxide nanoparticles were monitored online to obtain the particle size distribution using a scanning electrical mobility spectrometer (SEMS) [13]. The SEMS consists of an aerosol neutralizer ( $\mathrm{Kr}^{85}$ charger) to impart a known charge distribution to nanoparticles, a differential mobility analyzer (DMA, TSI 3081) to size charged nanoparticles, and a very sensitive electrometer (Keithley 6514A) to count nanoparticles size-selected by the DMA. Nanoparticles exiting the neutralizer, if charged, typically carry a single elementary charge due to their small sizes [14]. The DMA sizes charged particles according to their electrical mobilities [15]. For a given flow residence time, only particles having mobilities within a narrow range are transmitted through the DMA. The voltage applied to the DMA was scanned to probe the expected range of the particle size. A Faraday cage based on the design of Yun et al. [16] continuously collected all the singly charged particles selected by the DMA on a filter and the resulting current was measured by the Keithley electrometer. The particle concentration is then calculated by the ratio of the charge flow rate to the gas flow rate, taking into account the known charge distribution from the neutralizer and the transfer function of the DMA [13]. All devices and instruments were continuously monitored and controlled by a dedicated data acquisition (DAQ) computer running LabView, which displays the nanoparticle size distribution in a three-minute time interval.

A fraction of nanoparticles exiting the mini-arc plasma reactor was electrically charged by the plasma or thermionic emission of electrons from the nanoparticle surface. The charged nanoparticles were periodically collected for exsitu analyses by electrostatic precipitation $[17,18]$ onto carbon-coated TEM grids installed immediately downstream of the reactor. The TEM sample collection was initiated using a three-way valve. The collected particles were analyzed using a Hitachi H 9000 NAR TEM, which has a point resolution of $0.18 \mathrm{~nm}$ at $300 \mathrm{kV}$ in the phase contrast HRTEM imaging mode and is equipped with an energydispersive X-ray (EDX) spectrometer. Selected area diffraction (SAD) and amplitude contrast bright field (BF) imaging were also employed. The surface composition of as-produced 


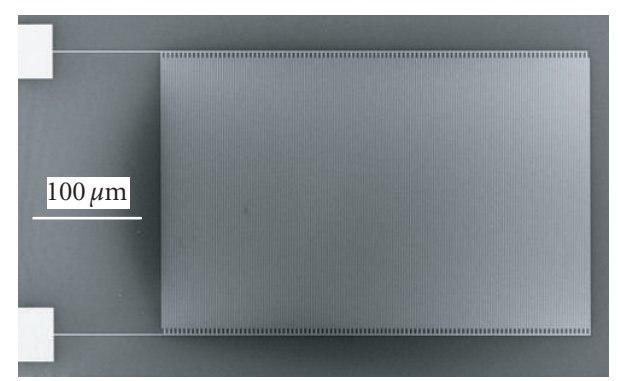

(a)

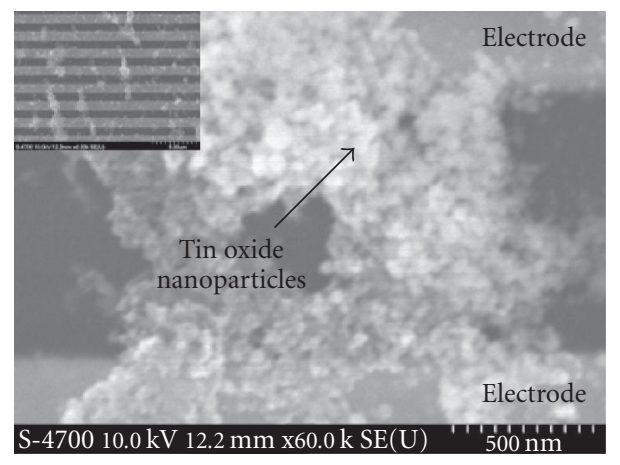

(b)

FIgURE 2: (a) SEM image of Au interdigitated electrodes used for sensor fabrication. The width and the separation of the electrodes are both $1 \mu \mathrm{m}$. (b) High resolution SEM image of a gas sensor fabricated by electrostatic assembly of tin oxide nanoparticles. The inset shows a larger area of the interdigitated electrode.

nanoparticles was analyzed using an HP5950 ESCA spectrometer with monochromatic $\mathrm{Al} \mathrm{K} \alpha$ radiation as $\mathrm{X}$-ray source.

The charged tin oxide nanoparticles from the miniarc nanoparticle generation system were assembled onto a prefabricated substrate with electrostatic force to build gas sensors. An interdigitated electrode based on the design of Kennedy et al. [5] was used to enable the electrostatic assembly of nanoparticles as well as the subsequent sensor characterization. The mechanism for functioning is simple; nanoparticles deposited between any two fingers close the electrical circuit to form a sensor, with the impedance of which changing in response to the exposed analyte molecules. For this purpose, an embedded gold interdigitated electrode in silicon substrate has been fabricated using e-beam lithography (30 KV Raith 150 e-beam tool) at Argonne National Laboratory (ANL). A scanning electron microscopy (SEM) image of the embedded electrode is shown in Figure 2(a). The line width of the gold electrode is $1 \mu \mathrm{m}$ and the spacing between the interdigitated fingers is also $1 \mu \mathrm{m}$. Figure 2(b) shows a high resolution SEM image of the final tin oxide nanoparticle sensor acquired on a Hitachi S4700 SEM at ANL. The inset shows a larger area of the interdigitated electrode bridged by tin oxide nanoparticles. The sensing performance of the nanoparticle sensor was evaluated at an operating temperature of $250^{\circ} \mathrm{C}$ with $100 \mathrm{ppm}$

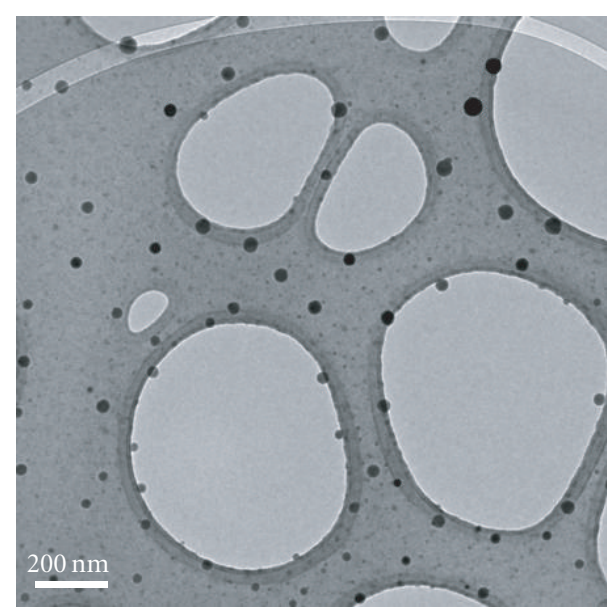

(a)

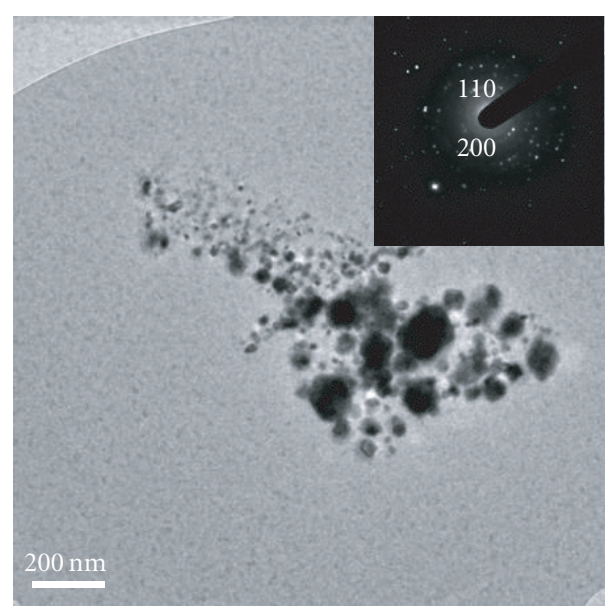

(b)

FIgURE 3: Bright field (BF) TEM images of tin oxide nanoparticles as produced from the mini-arc reactor. (a) Typical nonagglomerated nanoparticles are approximately spherical with diameter in the 20 to $40 \mathrm{~nm}$ range. (b) Agglomerated nanoparticles and nonspherical particles are seldom observed; the inset shows the selected area diffraction $(\mathrm{SAD})$ pattern of the particles.

ethanol vapor diluted in air. Resistance measurements were performed by applying a constant dc voltage $(10 \mathrm{~V})$ to the interdigitated circuit and monitoring the variation of the current passing through tin oxide nanoparticles when the sensor was exposed to either air or 100 ppm ethanol.

\section{RESULTS AND DISCUSSION}

Figure 3 shows two low-magnification bright field (BF) TEM images of the as-produced nanoparticles. Figure 3(a) is representative of the prevalent images with uniformly distributed and nonagglomerated nanoparticles that are preferred for gas sensor applications. The nonagglomerated entity of nanoparticles may be attributed to the fast coalescence of these particles in the high temperature environment. The larger particles shown in Figure 3(a) are approximately 


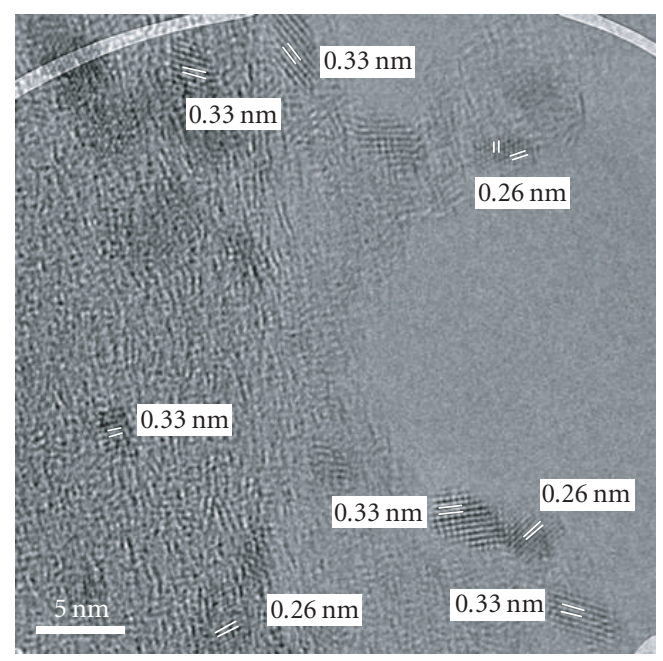

(a)

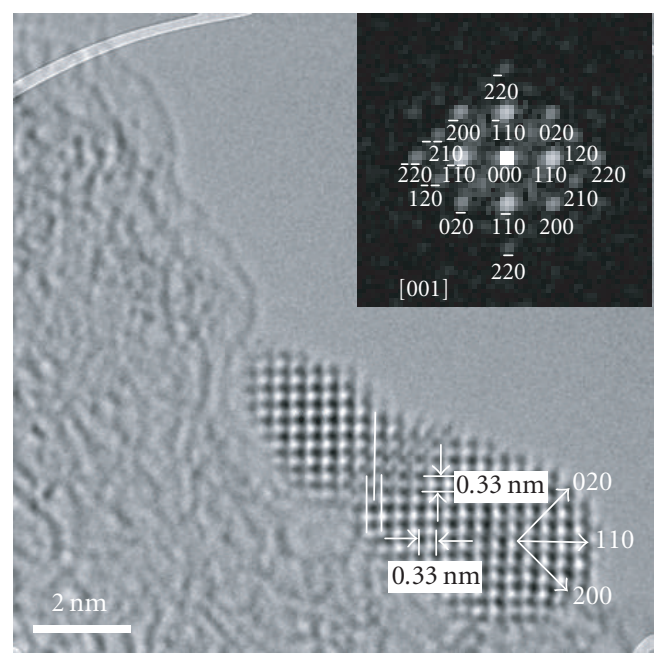

(b)

FIgURE 4: HRTEM images of (a) small nanoparticles showing lattice fringes corresponding to rutile $\mathrm{SnO}_{2}$ and (b) $\mathrm{SnO}_{2}$ agglomerate composed of two primary nanoparticles with denoted $\{110\}$ lattice fringes and $\{110\}$ and $\{200\}$ facet planes; the inset is the diffractogram of the agglomerate in the [001] projection and defect is observed at the joining neck where fringes misfit by $1 / 2(110)$.

spherical with diameters in the 20 to $40 \mathrm{~nm}$ range and slight ellipticity and/or small flat facets observed in some projections. This is a unique advantage of the mini-arc process since the high temperature in the arc reactor induces particle melting which leads to relatively large and at the same time quasi-spherical particles. Nanoparticles produced by many other vapor-phase processes, such as the flame spray synthesis [19], most often exhibit agglomerated structure if no special care is taken [20]. The smaller particles shown in Figure 3(a) can also be discerned, and are clearly shown in the HRTEM images of Figure 4. Although nonagglomerated nanoparticles are dominant, some agglomerated nanoparticles or agglomerates are also observed as shown in Figure 3(b). These agglomerates are likely formed due to the nonuniform residence time in the reactor. Although agglomerates are not preferred, their selected area diffraction (SAD) pattern shows that they are crystalline. With further examination, the unique reflections of (110) and (200) lattice planes of rutile $\mathrm{SnO}_{2}(a=4.74 \AA$ and $c=3.19 \AA)$ are identified.

HRTEM images of smaller $\mathrm{SnO}_{2}$ nanoparticles with sizes below $5 \mathrm{~nm}$ are shown in Figure 4(a). Lattice spacing analysis by numerical diffractograms gives recurrent values of 0.33 and $0.26 \mathrm{~nm}$, which correspond to the lattice spacings of rutile $\mathrm{SnO}_{2}$ from (110) and (101) reflections, respectively. Some agglomerates are also observed for these smaller particles. Figure 4(b) shows an agglomerate composed of two rutile $\mathrm{SnO}_{2}$ nanoparticles. The diffractogram shows that the zone axis of the diffraction pattern is [001]. Based on the indices of the diffraction pattern, three termination surfaces of the agglomerate are identified as (110), (020), and (200), with the (110) surface being reported as the lowest energy surface of rutile $\mathrm{SnO}_{2}$ [21]. This faceting may also explain the small flat regions seen at some of the edges of the larger round nanocrystals in Figure 3. Although both of the primary particles in Figure 4(b) are free of defects, fringes misfit by about $0.16 \mathrm{~nm}$ at the neck where the two particles join as a result of agglomeration. The defects present in the semiconductor $\mathrm{SnO}_{2}$ may adversely affect the density and transport of charge carriers, and consequently degrade the sensor performance [22]. As a result, agglomerated nanoparticles should be minimized to achieve the best sensor performance.

Elemental analysis from EDX (Figure 5(a)) clearly shows the presence of both $\mathrm{Sn}$ and $\mathrm{O}$ in the as-produced nanoparticle sample. However, the atomic ratio $\mathrm{O}: \mathrm{Sn}$ is below 2, which implies that not all as-produced nanoparticles are completely oxidized to stoichiometric $\mathrm{SnO}_{2}$. HRTEM images of smaller particles with a diameter of a few nm shown in Figure 4 already indicate that they are fully oxidized to $\mathrm{SnO}_{2}$. It is inferred that larger particles are not fully oxidized. The surface composition of the as-produced nanoparticles determined by XPS is shown in Figure 5(b). The binding energy for Sn $3 \mathrm{~d}_{5 / 2}$ peak at $486.9 \mathrm{eV}$ indicates the presence of $\mathrm{SnO}$ or $\mathrm{SnO}_{2}$ $[23,24]$. No metallic Sn peak (around $484.6 \mathrm{eV}$ [25]) was observed from the surface, which implies that the surface of larger nanoparticles is covered with an oxidation layer. Therefore, larger particles bear a core-shell structure, with a metallic core and an oxide shell. Since the binding energy peak $\mathrm{Sn} 3 \mathrm{~d}_{5 / 2}$ of $\mathrm{SnO}_{2}$ is very close to that of $\mathrm{SnO}[23,24]$, it is difficult to know the exact valence state of $\mathrm{Sn}$ in the surface oxidation layer. However, the binding energy of $486.9 \mathrm{eV}$ is relatively high compared to that of Sn, suggesting a larger fraction of higher valence state of Sn (IV) at the nanoparticle surface. The O $1 \mathrm{~s}$ peak shown in the XPS spectrum consists of two components, one at $530.5 \mathrm{eV}$ corresponding to oxygen bound to tin and the other at $532.1 \mathrm{eV}$ corresponding to adsorbed oxygen. The strong peak from adsorbed oxygen is attributed to the larger surface to volume ratio of smaller particles and suggests the enhanced sensing 


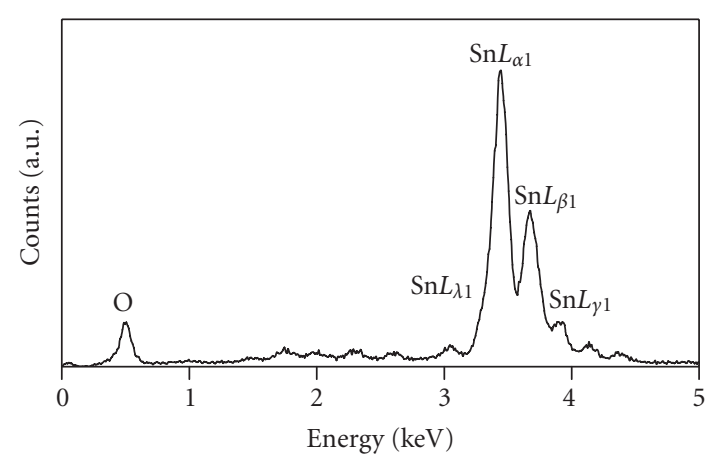

(a)

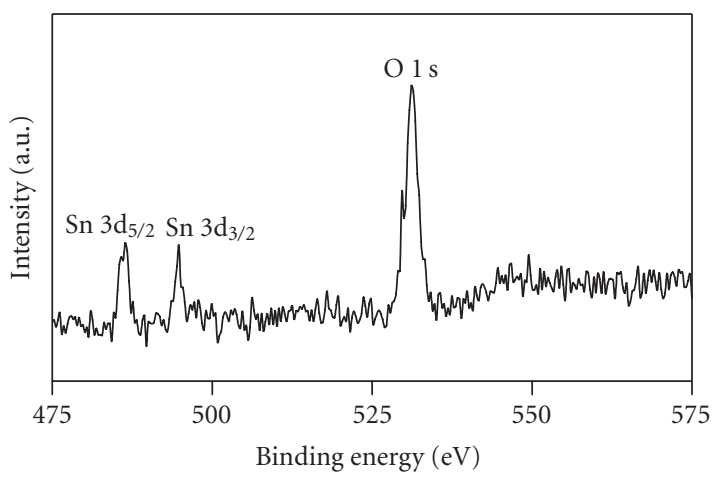

(b)

FIGURE 5: (a) EDX spectrum. (b) XPS spectrum of as-produced nanoparticles.

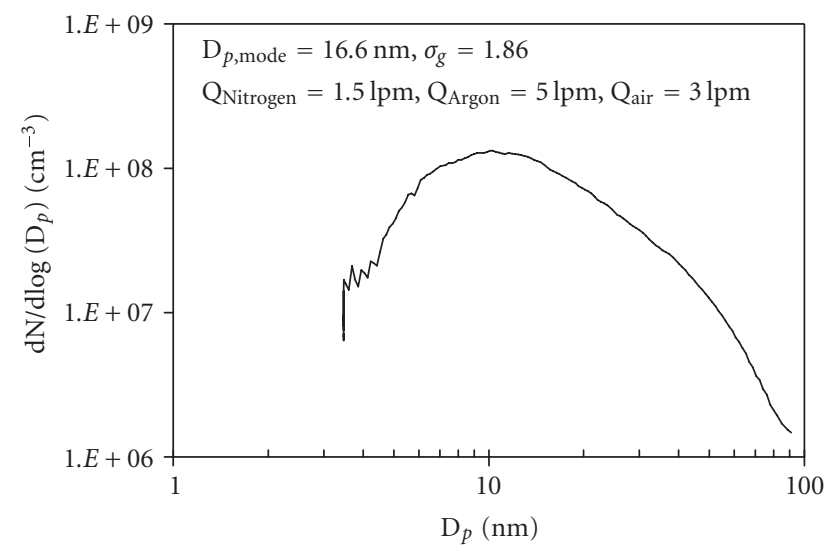

FIgURE 6: Tin oxide nanoparticle size distribution measured by SEMS for an arc current of $37.5 \mathrm{~A}$.

performance of these nanoparticles. After being heated in air for several hours before the sensor test, the particles are further oxidized. The sensing experiment presented later clearly shows that the surface $\mathrm{SnO}_{\mathrm{x}}$ layer on larger nanoparticles and the smaller $\mathrm{SnO}_{2}$ nanoparticles contribute to the rapid sensing reactions.

SEMS measurement of the size distribution of tin oxide nanoparticles used for gas sensor fabrication is shown in Figure 6 for an arc current of 37.5 A, an argon flow rate

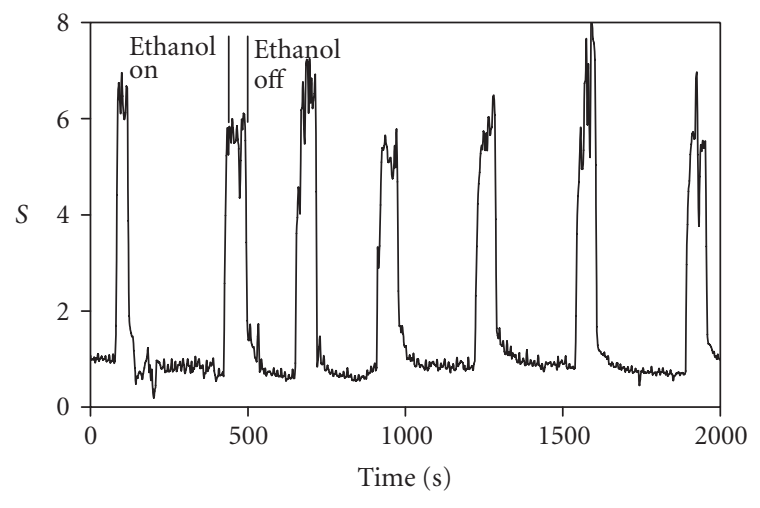

Figure 7: Dynamic response of tin oxide nanoparticle sensor to $100 \mathrm{ppm}$ ethanol at an operating temperature of $250^{\circ} \mathrm{C}$.

$\left(\mathrm{Q}_{\text {Argon }}\right)$ of $5 \mathrm{lpm}$, a nitrogen flow rate $\left(\mathrm{Q}_{\text {Nitrogen }}\right)$ of $1.5 \mathrm{lpm}$, and an air flow rate $\left(\mathrm{Q}_{\text {Air }}\right)$ of $3 \mathrm{lpm}$. The mode diameter $\left(\mathrm{D}_{p \text {,mode }}\right)$ of as-produced tin oxide nanoparticles is $16.6 \mathrm{~nm}$ and the geometrical standard deviation $\left(\sigma_{g}\right)$ is 1.86 . The size distribution of nanoparticles from the mini-arc plasma source may be adjusted by varying the quenching flow rate and other operating conditions. The quenching flow suppresses the coagulation of nanoparticles and slows down the particle growth. As a result, both $\mathrm{D}_{p \text {,mode }}$ and $\sigma_{g}$ of asproduced nanoparticle size distribution become smaller with the increase of quenching flow rate. Based on the SEMS measurements, the mass production rate of nanoparticles from the reactor was on the order of a few $\mathrm{mg} / \mathrm{hr}$. The evaporation rate of precursor tin was found to be on the same order of magnitude as the production rate by comparing the weight of precursor tin before and after the experiment. The reactor can potentially be scaled up by enabling a continuous supply of solid precursors and running many mini-arc sources in parallel. For fabrication of the gas sensor shown in Figure 2, the nanoparticle deposition time was about 30 minutes with a single reactor.

The small size and well-defined crystalline structure of as-produced tin oxide nanoparticles are expected to lead to improved gas sensing performance. Both the sensitivity and the dynamic response of the tin oxide nanoparticle sensor have been evaluated. The sensor sensitivity $\left(S=R_{a} / R_{g}\right)$ is defined as the ratio of the resistance of the sensor in air $\left(R_{a}\right)$ to the resistance in the sensing environment $\left(R_{g}\right)$. The dynamic response of the nanoparticle sensor is measured by recording the change in the resistance of the tin oxide nanoparticle sensor as a function of time when switching on or off the sensing gas. The time constant $(\tau)$ of the sensor is then determined by the time it takes for the sensor to attain $63.2 \%$ of the initial difference in electrical resistance before and after changing the gas environment.

Figure 7 shows the dynamic behavior of the tin oxide nanoparticle-based gas sensor at an operating temperature of $250^{\circ} \mathrm{C}$. The "ethanol on" represents the introduction of $100 \mathrm{ppm}$ ethanol and the "ethanol off" represents the introduction of clean air. Upon the introduction of ethanol, the 
resistance of the nanoparticle sensor decreases and a sensitivity greater than 1 is observed. Upon the introduction of clean air, the resistance of the nanoparticle sensor returns to a base value and a sensitivity around 1 is registered. The increased electrical conductivity of the tin oxide nanoparticle sensor is attributed to the reactions between ethanol and oxygen adsorbates on the surface of tin oxide nanoparticles that lead to the backflow of free electrons to tin oxide nanoparticles [26]. The sensor response is quite reproducible with an average sensitivity of about 6 . The fluctuation in sensitivity is most likely due to slight variations in the operating temperature and the gas flow rates during the experiment. The sensing time constant of the nanoparticle sensor is about 8 seconds while the recovery time constant is about 15 seconds. This sensing performance represents a significant improvement over that published in the literature. For example, Kennedy et al. [27] reported a tin oxide nanoparticle sensor with a sensitivity of 1.3, a sensing time constant of 110 seconds, and a recovery time constant of 403 seconds for the same operating conditions $\left(100 \mathrm{ppm}\right.$ ethanol and $\left.250^{\circ} \mathrm{C}\right)$. The higher sensitivity and faster response of the new sensor may be attributed to the smaller nonagglomerated tin oxide nanoparticles and smaller spacing between interdigitated fingers.

\section{CONCLUSION}

Tin oxide nanoparticles with sizes as small as a few $\mathrm{nm}$ have been successfully produced using a mini-arc plasma source to fabricate a functional gas sensor. Most of the larger nanoparticles greater than $20 \mathrm{~nm}$ are approximately spherical, while smaller nanoparticles below $5 \mathrm{~nm}$ are mostly faceted. Although some agglomerated nanoparticles are observed, nonagglomerated nanoparticles are dominant in the product. Based on the SAD and HRTEM analyses, both nonagglomerated and agglomerated nanoparticles bear a crystalline structure. However, agglomerated nanoparticles should be minimized for gas sensor applications since defects are induced at the joining neck as a result of agglomeration. Good response and sensitivity of the gas sensor fabricated from the as-produced tin oxide nanoparticles suggest that the mini-arc plasma source is capable of producing high-quality nanoparticles for gas sensing applications.

\section{ACKNOWLEDGMENTS}

The authors would like to thank Donald Roberson for technical support with TEM analyses, Liying Zhu and Steven Hardcastle for XPS analysis, and Nikolai Kouklin for assisting the initial sensor test. TEM analyses were performed in the HRTEM Laboratory at University of Wisconsin-Milwaukee (UWM) and XPS analyses were done at the Advanced Analysis Facility of UWM. The e-beam lithography was carried out in the Center for Nanoscale Materials at ANL and the high resolution SEM imaging was carried out in the Electron Microscopy Center at ANL, both of which are supported by the DOE Office of Science under contract \#W-31-109-Eng38. This work was financially supported by National Science Foundation (DMI-0609059) and UWM.

\section{REFERENCES}

[1] R. Summitt, J. A. Marley, and N. F. Borrelli, "The ultraviolet absorption edge of stannic oxide $\left(\mathrm{SnO}_{2}\right)$," Journal of Physics and Chemistry of Solids, vol. 25, no. 12, pp. 1465-1469, 1964.

[2] Y. Shimizu and M. Egashira, "Basic aspects and challenges of semiconductor gas sensors," MRS Bulletin, vol. 24, no. 6, pp. 18-24, 1999.

[3] D. E. Williams, "Conduction and gas response of semiconductor gas sensors," in Solid State Gas Sensors, P. T. Moseley and B. C. Tofield, Eds., p. 71, Adam Hilger, Bristol, UK, 1987.

[4] S. C. Chang, "Oxygen chemisorption on tin oxide: correlation between electrical conductivity and EPR measurements," Journal of Vacuum Science \& Technology, vol. 17, no. 1, pp. 366369, 1980.

[5] M. K. Kennedy, F. E. Kruis, H. Fissan, B. R. Mehta, S. Stappert, and G. Dumpich, "Tailored nanoparticle films from monosized tin oxide nanocrystals: particle synthesis, film formation, and size-dependent gas-sensing properties," Journal of Applied Physics, vol. 93, no. 1, pp. 551-560, 2003.

[6] M. Su, S. Li, and V. P. Dravid, "Miniaturized chemical multiplexed sensor array," Journal of the American Chemical Society, vol. 125, no. 33, pp. 9930-9931, 2003.

[7] E. R. Leite, I. T. Weber, E. Longo, and J. A. Varela, "A new method to control particle size and particle size distribution of $\mathrm{SnO}_{2}$ nanoparticles for gas sensor applications," Advanced Materials, vol. 12, no. 13, pp. 965-968, 2000.

[8] F. Gu, S. F. Wang, M. K. Lū, G. J. Zhou, D. Xu, and D. R. Yuan, "Photoluminescence properties of $\mathrm{SnO}_{2}$ nanoparticles synthesized by sol-gel method," Journal of Physical Chemistry $B$, vol. 108, no. 24, pp. 8119-8123, 2004.

[9] L. H. Jiang, G. Q. Sun, Z. H. Zhou, et al., "Size-controllable synthesis of monodispersed $\mathrm{SnO}_{2}$ nanoparticles and application in electrocatalysts," Journal of Physical Chemistry B, vol. 109, no. 18, pp. 8774-8778, 2005.

[10] F. E. Kruis, H. Fissan, and A. Peled, "Synthesis of nanoparticles in the gas phase for electronic, optical and magnetic applications - a review," Journal of Aerosol Science, vol. 29, no. 5-6, pp. 511-535, 1998.

[11] R. W. Siegel, "Synthesis and properties of nanophase materials," Materials Science and Engineering A-Structural Materials Properties Microstructure and Processing, vol. 168, no. 2, pp. 189-197, 1993.

[12] R. C. Flagan and M. M. Lunden, "Particle structure control in nanoparticle synthesis from the vapor phase," Materials Science and Engineering A-Structural Materials Properties Microstructure and Processing, vol. 204, no. 1-2, pp. 113-124, 1995.

[13] S. C. Wang and R. C. Flagan, "Scanning electrical mobility spectrometer," Aerosol Science and Technology, vol. 13, no. 2, pp. 230-240, 1990.

[14] A. Wiedensohler, "An approximation of the bipolar charge distribution for particles in the submicron size range," Journal of Aerosol Science, vol. 19, no. 3, pp. 387-389, 1988.

[15] D.-R. Chen, D. Y. H. Pui, D. Hummes, H. Fissan, F. R. Quant, and G. J. Sem, "Design and evaluation of a nanometer aerosol differential mobility analyzer (Nano-DMA)," Journal of Aerosol Science, vol. 29, no. 5-6, pp. 497-509, 1998.

[16] C.-M. Yun, Y. Otani, and H. Emi, "Development of unipolar ion generator-separation of ions in axial direction of flow," Aerosol Science and Technology, vol. 26, no. 5, pp. 389-397, 1997. 
[17] T. J. Krinke, K. Deppert, M. H. Magnusson, F. Schmidt, and H. Fissan, "Microscopic aspects of the deposition of nanoparticles from the gas phase," Journal of Aerosol Science, vol. 33, no. 10, pp. 1341-1359, 2002.

[18] J. Dixkens and H. Fissan, "Development of an electrostatic precipitator for off-line particle analysis," Aerosol Science and Technology, vol. 30, no. 5, pp. 438-453, 1999.

[19] T. Sahm, L. Madler, A. Gurlo, N. Barsan, S. E. Pratsinis, and U. Weimar, "Flame spray synthesis of tin dioxide nanoparticles for gas sensing," Sensors and Actuators, B: Chemical, vol. 98, no. 2-3, pp. 148-153, 2004.

[20] M. T. Swihart, "Vapor-phase synthesis of nanoparticles," Current Opinion in Colloid \& Interface Science, vol. 8, no. 1, pp. 127-133, 2003.

[21] E. R. Leite, T. R. Giraldi, F. M. Pontes, E. Longo, A. Beltrán, and J. Andrés, "Crystal growth in colloidal tin oxide nanocrystals induced by coalescence at room temperature," Applied Physics Letters, vol. 83, no. 8, pp. 1566-1568, 2003.

[22] X. Pan and J. G. Zheng, "Microstructure of and crystal defects in nanocrystalline tin dioxide thin films," in Proceedings of Materials Research Society Symposium (MRS '97), vol. 472, pp. 8792, Boston, Mass, USA, December 1997.

[23] P. A. Grutsch, M. V. Zeller, and T. P. Fehlner, "Photoelectron spectroscopy of tin compounds," Inorganic Chemistry, vol. 12, no. 6, pp. 1431-1433, 1973.

[24] H. Willemen, D. F. Van De Vondel, and G. P. Van Der Kelen, "An ESCA study of tin compounds," Inorganica Chimica Acta, vol. 34, pp. 175-180, 1979.

[25] R. I. Hegde, "Preparation of metal-polymer dispersions by plasma techniques: an ESCA investigation," Surface and Interface Analysis, vol. 4, no. 5, pp. 204-207, 1982.

[26] H. Fissan, M. K. Kennedy, T. J. Krinke, and F. E. Kruis, "Nanoparticles from the gas phase as building blocks for electrical devices," Journal of Nanoparticle Research, vol. 5, no. 3-4, pp. 299-310, 2003.

[27] M. K. Kennedy, F. E. Kruis, H. Fissan, and B. R. Mehta, "Fully automated, gas sensing, and electronic parameter measurement setup for miniaturized nanoparticle gas sensors," Review of Scientific Instruments, vol. 74, no. 11, pp. 4908-4915, 2003. 

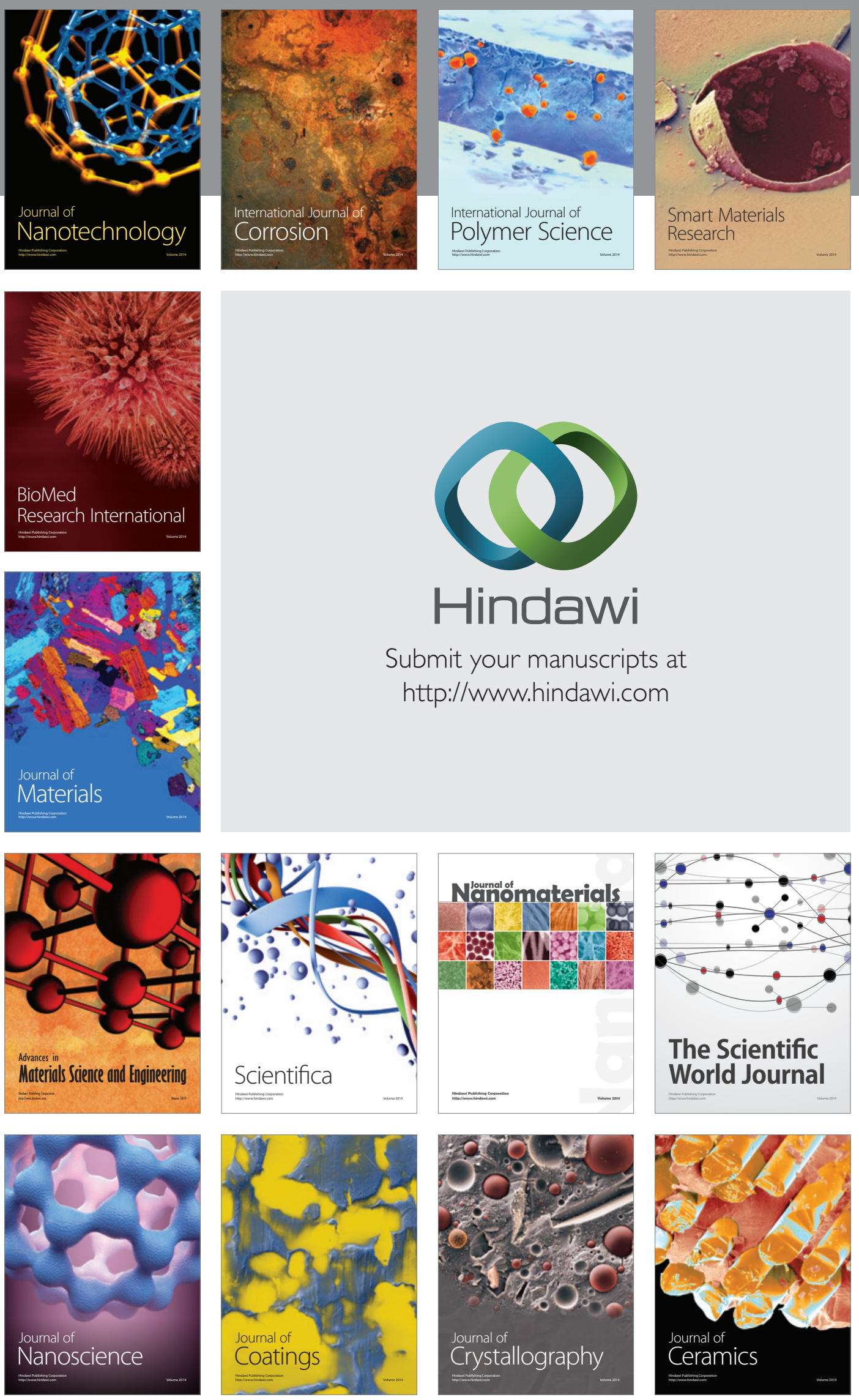

The Scientific World Journal

Submit your manuscripts at

http://www.hindawi.com

\section{World Journal}

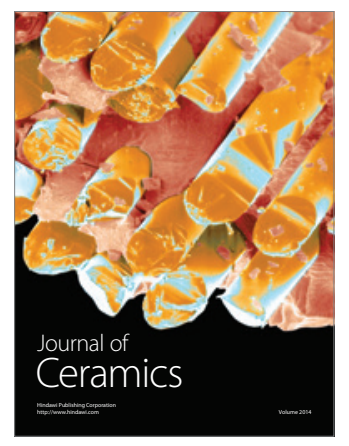

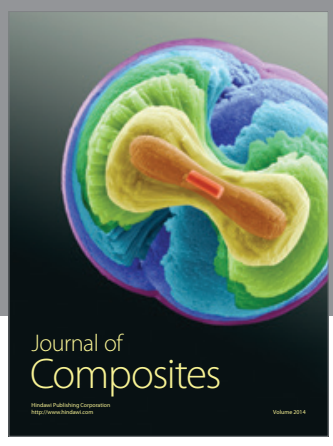
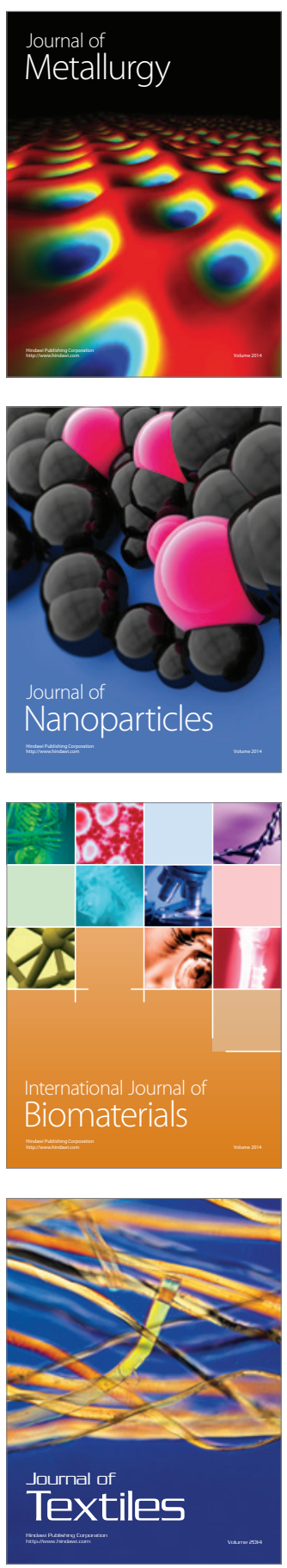